\title{
On-Chip Bus Arbiter Testing and Verification in FPGA
}

\author{
Rajesh Saha
}

\begin{abstract}
System-on-Chip (SoC) is the important key component for the integrated smart system design. All kinds of SoC are designed using different IP cores or functional block in silicon platform, which are inter-linked together by on chip bus communication architecture (OCBCA) to ensure optimum performance. Fairness to access and waiting time to access are very important characteristic for any arbiter. It's very difficult to analyze the performance of any arbiter accurately. Here we proposed an efficient methodology for testing and verification of onchip bus arbiter in FPGA environment. The primary objective is to determine path delay and fairness characteristics of arbiter components for On-chip bus of SoC by software Vivado-2013.4 environment and verified in the hardware Zynq (xc7z020) device of zed board environment.
\end{abstract}

Keywords

Arbiter Mechanisms;
FPGA;
On-chip bus of SoC;
System-on-Chip;
zynq-XC7Z020;

\section{INTRODUCTION}

The arbiter is operational components of $\mathrm{SoC}$ and, which are mainly acted as a decision maker component, which decides that which master component access the bus, when multiple masters are trying to access a shared or single bus. It is possible to integrate many functional cores can be connected through on-chip bus communication architectures (OCBCA) [1] on the same integrated circuits(IC), so that more parallel computation is possible [2]. Application domain of it is a system on a chip (SoC), which includes gaming system, automotive system, and a mobile terminal, etc. SoC design era believes reuse of the IP cores to the overall system design and attracts the designers to focus the functional operation and performance of the overall design. The performance of the design does not only depend on the OCBCA; it also depends on the logical arbitration component of the SoC.The logical arbiter component plays an important role in determining the performance of the SoC. Arbiter component which allows the processing elements based on few criteria, and these criteria are called arbitration mechanism [3]. As it assigns the priorities with which the processing blocks are granted to access a resource of the OCBCA. As increasing the integration of the component more would be the contention among the processing element for the bus hence to lead the violation of the real-time constraints, also degrade the performance of the system. As per example, it can limit the maximum number of bus cycles for which a master can use the bus through a maximum burst transfer size [4], or it can split transactions when slave devices are slow to respond to requests from a master. Few of conventional arbitration mechanisms are prioritybased and time division multiplexing (TDMA), round robin scheme, a hybrid of them, etc.. In the priority scheme, it has the disadvantage that is unfairness in access to the bus. Unfairness to access indicates that SoC component which has lower priority may get rare chance to get access to the bus [7]. This unfairness introduces the severe starvation of low priority master and results in extremely unfair bandwidth allocation hence reduced the overall performance of OCBCA [8]. There are many standards exist on chip bus which is working based on this methodology like AMBA, core connects [9], Wishbone, etc. To analyze performance parameter, tools, and methods are needed. In lottery bus, they used PTOLEMY software tool and methodology to analyze the performance of the arbiter component with the lottery algorithms. This paper describes the waiting time and testing environment of fairness check of design algorithms and performance comparison with new techniques and methodology. All these works carried in FPGA device Zynq-7z020 by the Vivado-2013.4 environment [10]. Rest of the paper is organized as follows: Section 2 Working principle of arbiter mechanism of OCBCA, Section 3 describes the experimental setup for evaluation of the performance of arbiter, Section 5 Results analysis and Section 6 concludes the paper.

\section{ARbITER MECHANISM}

Arbiters are electronic control mechanisms that allocate access to shared resources. It is used in the application of data packet switching and a bus arbiter. It's working based on some prior criteria, where users access the channel based on those standards. This approach is called algorithm of arbiter or arbitration scheme. An illustration the basic arbiter mechanisms for the on-chip bus are shown in Fig. 1. Initially arbiter component accepts the request signals from the entire masters then based on it criteria granted to the 
master. All the transactions are based on its control signal such address signal (ADDR), write data (WD) and control signal etc. are initiated by the master component read the slave components initiate read signal(RD) signals. Arbiter controls all these signals through three mux for different control signals of the component. Here memory mapped decoder is being used to select the slave-based control signals initiated by the master. These control signals depend on the interfacing protocol. Few standard protocols are advanced high bus protocol (AHB), advanced system bus protocol (ASB), advanced extension interface (AXI). Based on its high signal control highperformance data transfers mode support by bus standard like a pipeline, burst data transfer, split data transfer and out of order, etc.

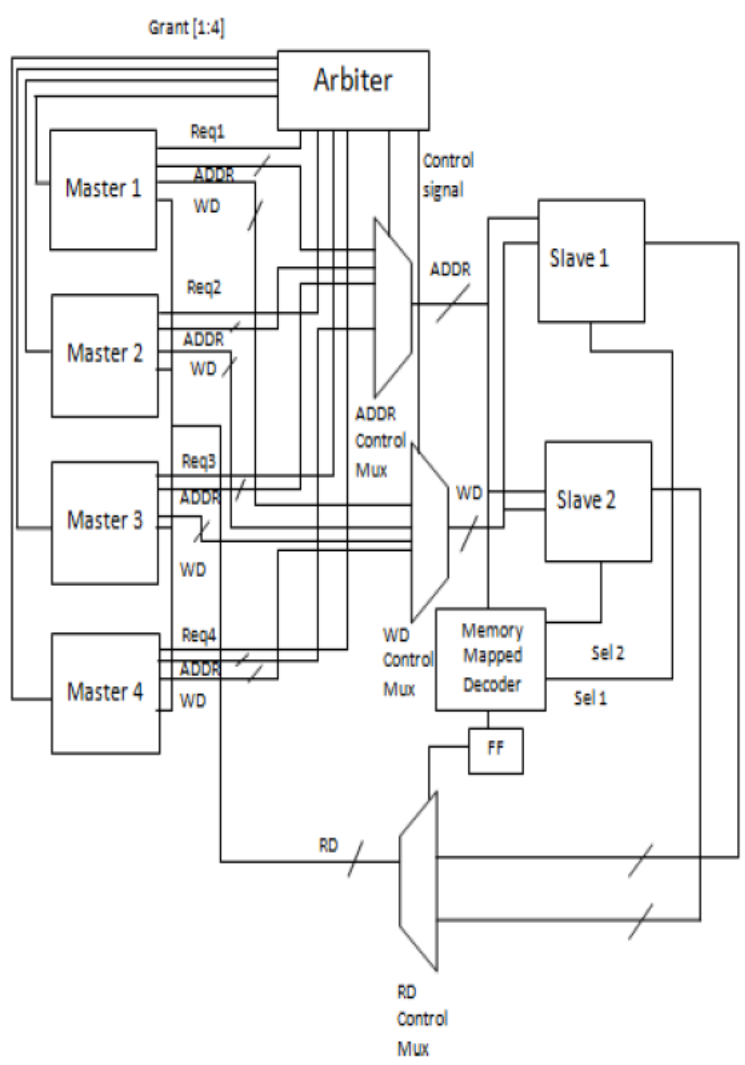

Fig 1. An illustration the basic arbiter mechanisms for the on-chip bus.

\section{EXPERIMENTAL SETUP FOR PERFORMANCE ANALYSIS}

Here we showed the hardware implementation of arbiter component in FPGA platform. FPGA is a programmable device which can reconfigure as per requirement. To do the entire task, we had taken
FPGA-based Zed board. It is a SoC development board consists of modules like FPGA Zynq device (xc7z020), ARM core and many input-output peripherals.

Here we used four DC signal to the input of the port by using the toggle switch of zed board and output signal passed through Pmod peripheral input-output port of the board with a clock signal $10 \mathrm{MHz}$ output signals are shown in DSO with respect to the clock signal.

Using Vivado design suite we calculated total delay in these logic circuits we get 147.32 ns for RRA.The performance of any arbiter is determined based on the waiting time or latency, fairness to access the bus [5]. Here we described that the experimental setup environment of the decision maker logics that is arbiter component. By this setup, we quickly analyze fairness to access to the bus and wait time of the arbiter. Waiting time of the design is the longest path or critical path of the design or total time required to do the transactions, fairness to the access to the bus means how many times, each master accesses the bus within the define cycle [11]. We have taken $4 * 4$ arbiter means four initiated signals from four different bus master to the arbiter with four grants signals to access the bus and counter is used to count the number of access of bus or channel by each master.

The output component observes counting of the counter. This test gives the experimental test of fairness access of the arbiter. For testing of waiting time, we initiated the signal by the master and that pass-through the design component and after few time of design, we get the output signal. Such type of methodology just connects oscilloscope probe to one at the input signal initiated by master and other at the output that is grant port of arbiter component, find the difference in its time scale. The arbiter mechanism are first initiated the signal by the master or any wave signal passed through the implemented arbiter on FPGA board of a device zynq-XC7Z020. Secondly, we have taken one signal from the output port of board and another from the input. After that, a signal from input ports and output ports of the arbiter component connects to the DSO and compares it's time axis to find the complete waiting path. We are taking as an example of round robin and fixed priority arbitration to check its fairness to access and waiting time or latency of the design system and compare its performance. For that, we are going to use the Verilog-HDL language in Xilinx Vivado-2013.4 platform to synthesize and verify as per the mentioned setup.

\section{Simulation Result Analysis}

Verilog-HDL language used to design and verification of its design. Vivado simulator used for simulation of proposed design. In fairness test or bandwidth utilization means how many times the each port can

Rajesh Saha, "On-Chip Bus Arbiter Testing and Verification in FPGA," International Journal of Advanced Engineering and Management, Vol. 2, No. 8, pp. 196-199, 2017.

DOI: https://doi.org/10.24999/IJOAEM/02080045 
access the channel. It means more access of channel gives information about the more contribution to use of bandwidth of the channel. Here all the ports access the channel equally except port1. But in priority scheme arbiter only highest priority port will access the whole bandwidth of the channel is shown in Fig 3.

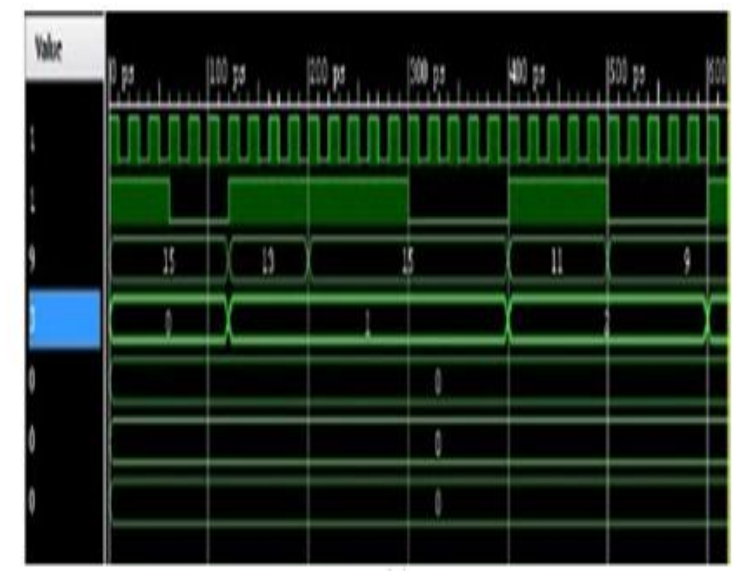

Fig 3. Simulation result for the arbiter scheme.

In each signal comes one after one, and that would be the waiting time

From these, it gives information that port 1 accesses the channel for whole time when all the ports busy with high traffic. Comparing both the figures. We can say RRA arbitration scheme is better than priority arbitration scheme in case of bandwidth utilization [12]. But for the case of area utilization, power consumption and architecture design of fixed priority arbiter is simpler than RRA.

\section{CONCLUSION}

Fairness to access and waiting time to access are very important characteristic for any arbiter. It's very difficult to analyze the performance of any arbiter accurately. The proposed software and hardware approach is useful to verify the functional and performance analysis of the logical arbiter component. For hardware verification, programmable device FPGA (xc7z020) fabric in zed board. We discussed arbitration scheme such as RRA and fixed priority and we do evaluate the performance of the arbitercomponents in software and hardware environment.

\section{REFERENCES}

[1] S Roy, R Saha, and C T Bhunia, "On Efficient Minimization Techniques of Logical Constituents and Sequential Data Transmission for Digital IC," Indian journal of science technology, vol. 9, no. 9, pp. 1-9, 2016.
[2] B Cordan, "An efficient bus architecture for system-on-a-chip design," in 12th Custom Integrated Circuits Conference, , USA, 1999, pp. 623-626.

[3] R Saha, V Nandi, S Roy, and C T Bhunia, "Design and verifications of efficient arbiter of SoC's on-chip bus," in Proceeding of IEEE 3rd International Conference on Electronics and Communication Systems, India, 2016, pp. 989992.

[4] Abdulsalam A Yayah, Yahaya Coulibaly, Abdul Samad Ismail, and George Rouskas, "Hybrid offset-time and burst assembly algorithm (H-OTBA) for delay sensitive applications over optical burst switching networks," International Journal of Communication System, pp. 1-11, 2014.

[5] K Lahiri, A Raghunathan, and G Lakshminarayana, "LOTTERYBUS: new communication architecture for high-performance system-on-chip designs," in Proceedings of 38th Design and Automation Conference, USA, 2001, pp. 15-20.

[6] Y S Cho, E J Choi, and K R Cho, "Modeling and analysis of the system bus latency on the SoC platform," in Proceedings of International Workshop SLIP, Germany, 2006, pp. 67-74.

[7] Josep Torrellas, Tucker Andrew, and Anoop Gupta, "Evaluating the performance of cacheaffinity scheduling in shared-memory multiprocessors," Journal of Parallel and Distributed Computing, pp. 139-151, 1995.

[8] D Flynn, "AMBA: Enabling reusable on-chip designs," IEEE Micro, vol. 17, no. 4, pp. 20-27, 1997.

[9] A Goel and R L William, "Formal verification of an IBM Core Connect processor local bus arbiter core," in Proceedings of ACM 37th Annual Design Automation, USA, 2000, pp. 196-200.

[10] Xilinx. (2016) ISE Synthesis and Verification Design Guide. [Online]. www.xilinx.com

[11] J. A Joao, "Bottleneck identification and acceleration in multithreaded applications (Doctoral dissertation).," 2014.

[12] K Padmanabham, Prabhakar Kanugo, M Chandrashekar, and K. Nagabhushan Raju, "MILSTD-1553 bus protocol algorithms implementation on fpga to realise system-on-chip

Rajesh Saha, "On-Chip Bus Arbiter Testing and Verification in FPGA," International Journal of Advanced Engineering and Management, Vol. 2, No. 8, pp. 196-199, 2017.

DOI: https://doi.org/10.24999/IJOAEM/02080045 
(SOC)," International Journal of Advancements in Research \& Technology, vol. 5, no. 5, pp. 1-8, 2016.

Author

Rajesh Saha

Project Engineer,

Department of Electronics Engineering

NIT Uttarakhand, Srinagar Garhwal

rajeshsaha29@gmail.com

Editor-in-Chief: Sahadev Roy

Rajesh Saha, "On-Chip Bus Arbiter Testing and Verification in FPGA," International Journal of Advanced Engineering and Management, Vol. 2, No. 8, pp. 196-199, 2017.

DOI: https://doi.org/10.24999/IJOAEM/02080045 